\title{
Algorhythm for Use of Percutaneous Short Fixation of Fractures Involving the Thoracolumbar Junction and Lumbar Spine
}

\author{
Nicola Marotta, Alessandro Landi*, Roberto Delfini \\ Neurosurgery Department of University of Rome Sapienza, Rome, Italy. \\ Email: *dott.alessandro.landi@gmail.com \\ Received April 12 $2^{\text {th }}, 2013$; revised May $5^{\text {th }}, 2013$; accepted May $15^{\text {th }}, 2013$ \\ Copyright (C) 2013 Nicola Marotta et al. This is an open access article distributed under the Creative Commons Attribution License, \\ which permits unrestricted use, distribution, and reproduction in any medium, provided the original work is properly cited.
}

\begin{abstract}
Study Design: Original article. Objective: Guidelines for deciding whether to perform open or percutaneous surgery in burst fractures. Summary of Background Data: The authors propose an algorithm for deciding whether to perform open surgery or percutaneous surgery with short fixation in patients with fractures of the thoracolumbar junction and lumbar spine. Methods: Between July 2005 and July 2009, 72 patients underwent surgical stabilization by posterior route for fractures of the thoracolumbar junction and lumbar spine. In 44 the lesion involved the thoracolumbar junction, in 28 the lumbar spine (L2 in 6 cases, L3 in 15 cases, L5 in 7 cases). The fractures were assessed morphologically according to Magerl's classification (52 type A, 12 type B, 8 type C). All patients were analyzed according to the algorithm proposed, according to which patients must fulfil certain criteria: the fracture must be Magerl type A.3, it must involve one level, McCormack score must be 6 or less, invasion of the spinal canal must be $25 \%$ or less according to Hashimoto's formula, Magnetic Resonance Imating (MRI) must confirm discoligamentous integrity. Neurologically, the patient must be ASIA E. 25 patients (17 thoracolumbar junction, 8 lumbar spine) fulfilled these criteria and were treated by percutaneous short fixation. Results: The average length of the surgical procedure was 80 minutes and the loss of blood $10 \mathrm{cc}$. All patients were dismissed without brace and were submitted to follow-up Computed Tomography CT scan 3 and 6 months after surgery. Follow-up ranged from 6 months to 4 years. In all cases CT scan confirmed fusion and there were no cases of rupture of the device. None of the patients presented neurological deficits. Conclusion: The algorithm described permits a proper selection of patients with thoracolumbar fractures who can be treated by percutaneous short fixation, thus avoiding the risks connected with failure of the stabilization system.
\end{abstract}

Keywords: Minimally Invasive Spine Surgery; Percutaneous Short Fixation; Thoraco-Lumbar Fractures

\section{Introduction}

Over the years minimally-invasive spinal surgery has become more and more important and its application in traumatic spinal injuries is a logical step forward. Since the choice of treatment for fractures of the thoracolumbar junction and lumbar spine is the topic of much debate both in the literature and in daily practice, the authors have devised an algorithm for selecting which patients should be treated by percutaneous short fixation for fractures of thoracolumbar junction and lumbar spine. Our algorithm is based on: fracture's morphology in according with Magerl's classification [1], one level fracture, load sharing classification in according with McCormack

*Corresponding author. scale [2], invasion of the spinal canal in according with Hashimoto formula [3], integrity of posterior discoligamentous complex evaluated by MRI scans, preoperative neurological status in according with ASIA scale.

\section{Materials and Methods}

Between July 2005 to July 2009, 72 patients underwent surgery for thoraco-lumbar and lumbar fractures in the Neurosurgery division of "Sapienza" University of Rome (Table 1). All these patients underwent diagnostic imaging including CT scans, X-rays scans in Anterior/Posterior and Latero/Lateral projections, and MRI scans. In all of them the algorithm, indicating the type of surgical approach, ranging from percutaneous with short fixation to open surgery, was applied (Figure 1). Of these, 
Table 1. Historic cohort: demographic data, morphology of the fractures, detailed analysis of algorithm parameters.

\begin{tabular}{|c|c|c|c|c|c|c|c|c|c|c|c|c|c|}
\hline & Sex & Age & Level & $\begin{array}{c}\text { Magerl } \\
\text { class }\end{array}$ & $\begin{array}{l}\text { McCormack } \\
\text { score }\end{array}$ & $\begin{array}{c}\text { Invasion } \\
\text { Hashimoto } \\
\text { formula }\end{array}$ & ASIA & $\begin{array}{c}\text { MRI } \\
\text { pls }\end{array}$ & Surgery & Ligamentotaxis & Reduction & $\begin{array}{c}\text { Consolidation } \\
\text { time at CT } \\
\text { follow-up }\end{array}$ & Smoker \\
\hline 1 & M & 33 & $\mathrm{~T} 12$ & A. 3.3 & 6 & $23 \%$ & $\mathrm{E}$ & YES & T11-L1 & YES & Complete & 3 months & - \\
\hline 2 & M & 30 & L2 & A. 3.1 & 4 & $\mathrm{~N}$ & $\mathrm{E}$ & YES & L1-L3 & - & - & 3 months & Yes \\
\hline 3 & M & 24 & $\mathrm{~T} 12$ & A.3.1 & 4 & $\mathrm{~N}$ & E & YES & T11-L1 & - & - & 3 months & - \\
\hline 4 & $\mathrm{~F}$ & 44 & L1 & A. 3.3 & 4 & $20 \%$ & E & YES & T12-L2 & YES & Complete & 6 months & Yes \\
\hline 5 & M & 20 & $\mathrm{~T} 12$ & A. 3.2 & 3 & $\mathrm{~N}$ & $\mathrm{E}$ & YES & T11-L1 & - & - & 3 months & - \\
\hline 6 & $\mathrm{~F}$ & 29 & $\mathrm{~L} 2$ & A. 3.3 & 5 & $25 \%$ & E & YES & L1-L3 & YES & Partial & 3 months & - \\
\hline 7 & M & 36 & L5 & A.3.1 & 3 & $\mathrm{~N}$ & $\mathrm{E}$ & YES & L4-S1 & - & - & 3 months & - \\
\hline 8 & M & 40 & L1 & A. 3.2 & 4 & $\mathrm{~N}$ & E & YES & T12-L2 & - & - & 3 months & - \\
\hline 9 & M & 49 & L3 & A.3.1 & 3 & $\mathrm{~N}$ & E & YES & T12-L2 & - & - & 6 months & Yes \\
\hline 10 & M & 22 & L1 & A. 3.2 & 5 & $14 \%$ & E & YES & L2-L4 & YES & Complete & 3 months & - \\
\hline 11 & $\mathrm{~F}$ & 29 & L1 & A. 3.1 & 3 & $\mathrm{~N}$ & E & YES & T12-L2 & - & - & 3 months & Yes \\
\hline 12 & M & 38 & $\mathrm{~T} 12$ & A. 3.2 & 5 & $10 \%$ & E & YES & T11-L1 & YES & Partial & 3 months & Yes \\
\hline 13 & M & 30 & L1 & A. 3.1 & 3 & $\mathrm{~N}$ & E & YES & T12-L2 & - & - & 3 months & - \\
\hline 14 & M & 41 & L5 & A. 3.2 & 4 & $17 \%$ & $\mathrm{E}$ & YES & L4-S1 & YES & Complete & 3 months & Yes \\
\hline 15 & M & 41 & L5 & A.3.1 & 3 & $\mathrm{~N}$ & E & YES & L4-S1 & - & - & 3 months & - \\
\hline 16 & $\mathrm{~F}$ & 54 & L1 & A.3.1 & 3 & $\mathrm{~N}$ & E & YES & T12-L2 & - & - & 6 months & Yes \\
\hline 17 & $\mathrm{~F}$ & 42 & L1 & A. 3.2 & 3 & $\mathrm{~N}$ & E & YES & T12-L2 & - & - & 3 months & - \\
\hline 18 & M & 24 & L3 & A. 3.2 & 3 & $\mathrm{~N}$ & $\mathrm{E}$ & YES & L2-L4 & - & - & 3 months & - \\
\hline 19 & M & 37 & $\mathrm{~T} 12$ & A. 3.3 & 5 & $25 \%$ & $\mathrm{E}$ & YES & T11-L1 & YES & Complete & 3 months & - \\
\hline 20 & M & 36 & L3 & A. 3.2 & 4 & $8 \%$ & E & YES & L2-L4 & YES & Complete & 3 months & - \\
\hline 21 & $\mathrm{~F}$ & 37 & $\mathrm{~T} 12$ & A. 3.2 & 3 & $\mathrm{~N}$ & E & YES & T11-L1 & - & - & 3 months & - \\
\hline 22 & $\mathrm{~F}$ & 52 & L1 & A.3.1 & 3 & $\mathrm{~N}$ & E & YES & T12-L2 & - & - & 6 months & Yes \\
\hline 23 & $\mathrm{~F}$ & 50 & L1 & A.3.1 & 2 & $\mathrm{~N}$ & E & YES & T12-L2 & - & - & 6 months & Yes \\
\hline 24 & M & 41 & L1 & A. 3.3 & 6 & $22 \%$ & E & YES & T12-L2 & YES & Complete & 3 months & - \\
\hline 25 & M & 47 & $\mathrm{~T} 12$ & A. 3.2 & 3 & $\mathrm{~N}$ & E & YES & T11-L1 & - & - & 6 months & Yes \\
\hline
\end{tabular}

according to Magerl's classification, 52 belonged to group A, 12 to group B and 8 to group C. In accordance with our algorithm, 25 cases were selected (17 males$68 \%$, and 8 females- $32 \%$, with an average age of 37.04 years) as suitable for percutaneous short fixation. Of this group there was a fracture of the junction in 17 patients$68 \%$ (10 males and 7 females) while the remaining 8$32 \%$ ( 7 males and 1 female) had a lumbar fracture. The vertebral level affected by the fracture in the selected cohort was T12 in 7 cases (28\%), L1 in 10 cases (40\%), L2 in 2 cases $(8 \%), \mathrm{L} 3$ in 3 cases $(12 \%)$ and L5 in 3 cases (12\%). All fractures selected are type A.3 in according to Magerl's classification. Of these, there are 10 fractures type A.3.1 (40\%), 10 fractures type A.3.2 (40\%) and 5 fractures type A.3.3 (20\%). On preoperative MRI scans the disco-ligamentous complex always appeared to be integral; Using the Hashimoto formula, we have shown that 9 of these cases presented $\leq 25 \%$ of spinal canal invasion (36\%) whereas $16(64 \%)$ did not present any invasion of the canal (Figure 2). The ASIA scale showed that all patients were neurologically intact. The McCormack Score in these 25 patients was $\leq 6$ (range 2 6). These 25 cases were treated by percutaneous surgery with short fixation. One patient with a T12 A.3.2 fracture also presented a Hangman type 1 fracture of $\mathrm{C} 2$ that was treated conservatively using a Philadelphia collar as well as a fracture of the hip, for which she was transferred to an orthopedic division immediately after T11-L1 stabili- 


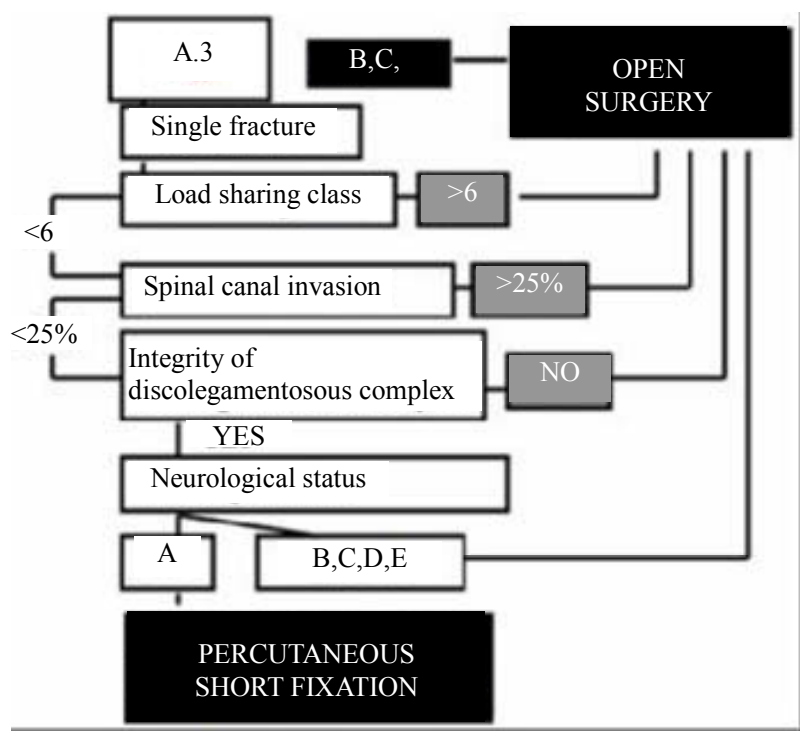

Figure 1. The algorithm purposed by our institute based on: fracture's morphology in according with Magerl's classification, one level fracture, load sharing classification in according with McCormack, invasion of the spinal canal in according with Hashimoto formula, integrity of posterior discolegamentous complex evaluated by MRI scans, preoperative neurological status in according with ASIA scale.

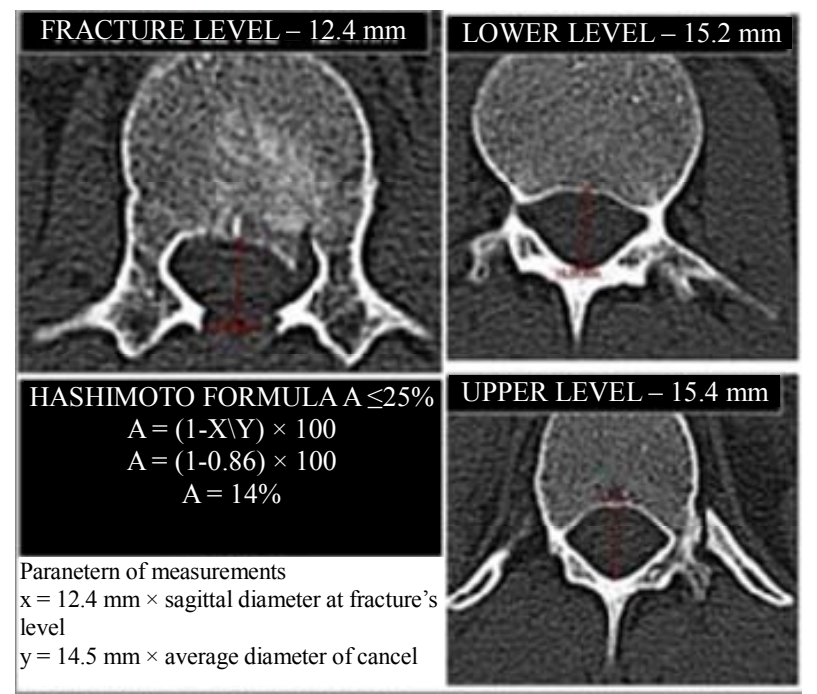

Figure 2. Application of Hashimoto's formula in the descriptive case.

zation, for surgical treatment. In the 9 patients with narrowed canal, an intraoperative reduction of the fracture by distraction and ligamentotaxis was performed. Follow-up lasted from 6 months to 4 years. In all patients early postoperative neuroimaging control images were obtained by $\mathrm{CT}$ and $\mathrm{x}$-rays scans, repeating CT-scans at 3 and 6-month follow-up. All patients were able to walk in first postoperative day and were dismissed without any type of brace in second postoperative day. For all the patients, was proposed the removal of the implant after the fracture's consolidation, which as to be done not over 6 - 8 months after surgical procedure.

\section{Descriptive Case}

A 22-year-old man, falls from height, came to our hospital with acute low back pain, without any neurological deficits (ASIA E). He underwent CT and MRI (Figures 3(a)-(c)) in emergency that showed a L1 burst fracture, type A.3.2, with integrity of posterior ligaments. McCormack score was 5 and measurements of Hashimoto formula showed a spinal canal invasion of $14 \%$ (Figure 2). He underwent surgical treatment with posterior percutaneous short fixation at T12-L2, with complete reduction of the fracture performing by ligamentotaxis (Figures 4(a)-(e)). He began to walk without corset on the $1^{\text {st }}$ postoperative day and he was discharged in second postoperative day without brace. CT scan performed 3 months after surgery showed a complete fusion of the fracture. He underwent surgical removal of the implant 6 months after surgery without any complications (Figures 3 and 4).

\section{Results}

All patients underwent treatment within 48 hours of the traumatic event. Surgical operation lasted about $80 \mathrm{~min}$ utes with an average intraoperative blood loss of $10 \mathrm{cc}$. In the group of 9 patients in which was performed reduction by distraction and ligamentotaxis, we have 7 cases in which the reduction was complete and 2 cases in which was partial and there weren't any clinical sign during follow-up. All patients had an optimal postoperative

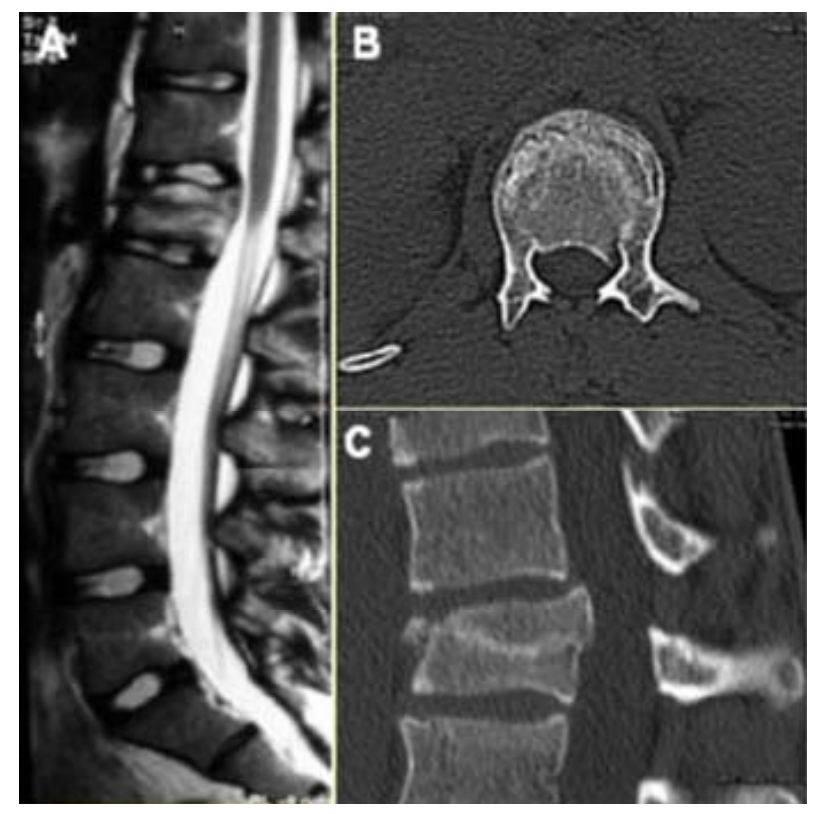

Figure 3. (a) Preoperative MRI: sagittal T2 weighted images; (b) and (c) Sagittal and axial preoperative CT scans. 


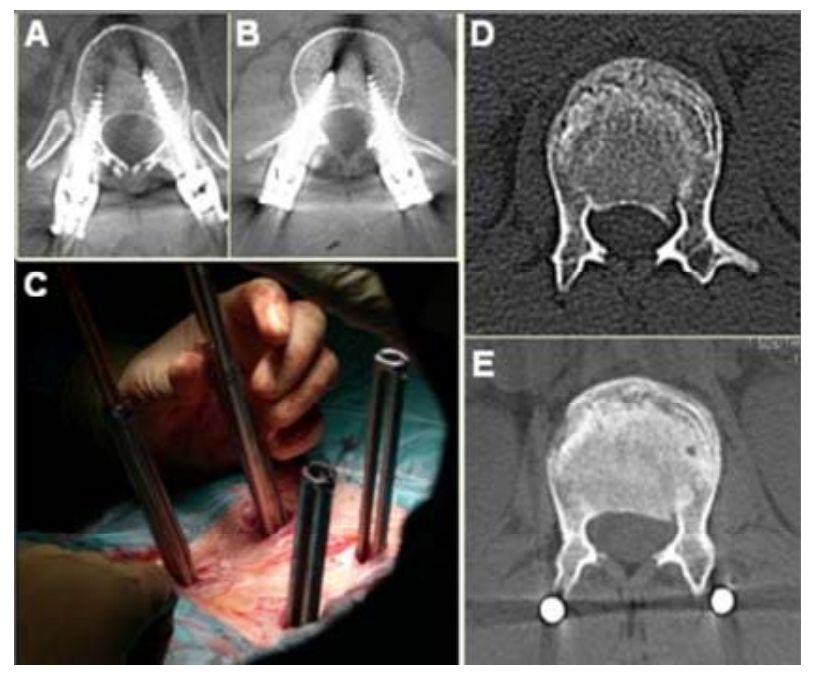

Figure 4. (a) and (b) Postoperative CT scans that shows the correct screws placement; (c) Intraoperative visualization of tubular dilatators form which the screws was inserted; (d) and (e) Comparison between preoperative and postoperative CT scans at the level of the fractures, that sowed the complete reduction performed by ligamentotaxis.

course and began to walk without corset on the $1^{\text {st }}$ postoperative day prior to discharge on day 2. In the CT scans performed after 6 months, all cases showed a consolidation of the fracture. Moreover, it was evidenced that in 19 cases $(76 \%)$ the consolidation was complete after 3 months. The 6 consolidated cases after six months (24\%) were smokers (mean age $49.3 \mathrm{yrs}$ ), and 4 smokers consolidated after 3 months (mean age $34.5 \mathrm{yrs}$ ). None of them have presented neurological worsening or postoperative pain. In fact, late functional and neurological evaluation with CT scan has confirmed fusion and ruled out any breakage of the implant. Only 17 patients (68\%) underwent surgical removal of the implant after consolidation of the fracture without any neurological deficits and any complications. No neurological deficits were observed throughout the follow-up period.

\section{Discussion}

Minimally-invasive spinal surgery was initially devised for the treatment of degenerative spine pathology and has become more and more popular over the years [4-8]. The idea of being able to perform minimally-invasive or percutaneous stabilization of thoracolumbar fractures is extremely appealing owing to the well-known advantages of this type of surgery (less blood loss and postoperative pain, less need for pain-killing drugs and shorter operating times) and to the increased frequency of short fixation at thoracolumbar level [8]. The indication for short fixation at the level of the thoracolumbar junction and lumbar spine is still a much debated topic in the literature: on one hand many authors advocate this choice for its low biomechanical impact on the spine while, on the other hand, other authors emphasize the possibility of a high failure rate [9-11]. In our opinion, minimally-invasive surgery could be optimally employed for short fixation since it fulfills the requirements of this type of treatment. The purpose of the decisional algorithm is to guide the surgeon in his choice between a percutaneous approach and open surgical instrumentation: its limits consist of the presence of other contiguous spinal fractures, the morphological features of the fracture, the integrity of the disc and ligaments, the amount of spinal canal invasion and the patient's neurological status (Figure 2). It is clear that, before considering a short fixation, other adjacent fractures that would put any stabilization ending in a fractured vertebral body at great risk must be excluded: it would be equally risky to have the segment adjacent to the end of the fixation with a fracture, even if this does not require surgery, owing to the stress that the implant would exert on it. The morphology of the fracture visualized by CT scan is fundamental for an initial classification of the fracture according to Magerl so that in type B and type $\mathrm{C}$ lesions percutaneous treatment can be excluded $[1,12,13]$. For type B fractures, in which decompression is not necessary, a far lateral minimally-invasive approach can be performed: this choice allows to preserve the insertion of paravertebral muscles on laminae and spinous processes, preserving the biomechanical function; moreover is possible to perform a posterolateral fusion, that is necessary in this type of fracture. This decision is made taking into consideration both the high grade of instability attributed to lesions that occur via a flexion-extension or rotation mechanism which present bone and/or ligamentous damage such as lesions of the disc and the posterior ligamentous complex, as well as the fact that this is only an initial experience and it is likely that the algorithm will be modified as our experience increases and improves. In this way, the only fractures included in the algorithm are those resulting from axial loading (type A fractures) that can be well-diagnosed by CT scan. Of type A fracture, the A.1 and A.2 group have a very low rate of instability and possibility of kyphosis evolution, so these type of fractures have a complete recovery with conservative treatment, with rigid or semirigid corset. Up to us, algorithm can be applied only for the type A.3 burst fractures and their subtype. Furthermore, CT imaging makes it possible to address 2 aspects of the algorithm, namely the classification of the fracture according to McCormack's Load Sharing classification and the amount of spinal canal involvement according to Hashimoto's formula [2,3]. A McCormack score of 6 or less is predictive of a successful short fixation. In our opinion, a percutaneous approach can be safely employed with an extremely low risk of neurological deficits in cases presenting up to $25 \%$ of spinal 
canal invasion, calculated using Hashimoto's formula: beyond this degree of invasion the risks involved are far more significant $[14,15]$. It is well-known that the amount of kyphosis and the amount of correction obtainable represent important parameters in McCormack's rating: percutaneous systems make it possible to correct kyphosis and ligamentotaxis using excellent distraction and compression systems, thus reducing the amount of spinal canal invasion. Moreover, MRI evaluation of the disc and posterior ligamentous complex provides further data regarding stability: therefore, in the event of these two structures presenting signs of injury, the treatment of choice shouldn't be percutaneous surgery because the posterior fusion must be perform, in association with a long fixation if necessary. Vaccaro added the integrity of the posterior ligamentous complex to the factors that play an important role in the treatment of thoracolumbar fractures, considering it to be a discriminating factor for the choice of surgical approach together with neurological status [16]. The last parameter considered in the algorithm is, indeed, neurological status. In fact, only patients with an E rating on the ASIA classification are eligible for percutaneous stabilization whereas in all the others open surgery is performed to allow decompressive laminectomy that, in our opinion, is mandatory even in paraplegic patients, in order to prevent secondary ischemic damage as described by Vaccaro [17], although some authors believe that there isn't any relationship between spinal canal invasion and neurological status [15]. In our experience, the difficulties encountered in treatment of thoracolumbar fractures derived from the fact that this is still a much debated topic in the literature; in fact, for each single case there are a myriad of different morphological, biomechanical and therapeutic interpretations $[14,17]$. There is also a real risk of erroneously choosing to perform percutaneous treatment for a thoracolumbar fracture because the enthusiasm understandably evoked by this type of treatment can influence the choice of the most suitable treatment option for the type of fracture involved [17]. The algorithm described here was devised and applied to fulfill the need for a correct indication for percutaneous treatment, (not susceptible to individual interpretation). The results obtained were positive since all the patients selected and treated mini-invasively using the algorithm presented CT confirmation of fusion without neurological deficits and were able to return promptly to their previous daily activities. This procedure allows to an early mobilization of patients without brace, resulting in a rapid and comfortable postoperative functional recovery, especially if compared with cases treated conservatively, that are described in literature, where it was necessary to wear a brace for at least 4 months. Moreover the early recruitment upright without brace reduce the risk of muscle's atrophy caused by the pro- longed wearing of brace, often responsible of pain and functional biomechanical limitations in patients treated conservatively. Moreover, in one patient with multiple traumatic injuries of the hip and lower limbs, percutaneous treatment made it possible for the patient to be submitted earlier to the next multi-specialty phase of treatment, consisting of orthopedic surgery and motor rehabilitation, thus considerably shortening recovery times. In the light of this, we can safely say that the algorithm can be employed in multiorgan injured patients since it reduces operative risks and allows earlier patient mobilization. Other important aspect for which percutaneous stabilization was preferred, is the possibility of future removal of the implant to preserve motility of the instrumented segment as demonstrated by Yurec et al. too [18]. This removal is extremely simple because the surgical approach and the instrumentation are the same of the fixation.

\section{Conclusion}

The algorithm described permits a proper selection of patients with thoracolumbar fractures suitable for percutaneous short fixation, thus avoiding the risks consequent to eventual breakage of the stabilization system. The algorithm described can be used as a selective parameter even in patients with multiple trauma, allowing earlier patient mobilization and shortening the time needed to achieve multi-specialty therapeutic programs.

\section{REFERENCES}

[1] F. Magerl, "A Comprehensive Classification of Thoracic and Lumbar Injuries," European Spine Journal, Vol. 3, No. 4, 1994, pp. 184-201. doi:10.1007/BF02221591

[2] T. McCormack et al., "The Load Sharing Classification of Spine Fractures," Spine, Vol. 19, No. 15, 1994, pp. 1741-1744. doi:10.1097/00007632-199408000-00014

[3] T. Hashimoto, "Relationship between Traumatic Spinal Canal Stenosis and Neurologic Deficits in Thoracolumbar Burst Fractures," Spine, Vol. 13, No. 11, 1988, pp. 12681272. doi:10.1097/00007632-198811000-00011

[4] W. Caspar, "A New Surgical Procedure for Lumbar Disc Herniation Causing Less Tissue Damage through a Microsurgical Approach," Advanced Neurosurgery, Vol. 4, 1977, pp. 74-80. doi:10.1007/978-3-642-66578-3_15

[5] S. Hijigata, et al., "Percutaneous Diskectomy: A New Treatment Method for Lumbar Disc Herniation," Journal of Toden Hospital Vol. 5, 1975, pp. 5-13.

[6] L. T. Khoo, "Minimally Invasive Percutaneous Posterior Lumbar Intebody Fusion," Neurosurgery, Vol. 51, No. 5S, 2002, pp. S166-S181.

[7] A. Muller, "A Keyhole Approach for Endoscopically Assisted Pedicle Screw Fixation in Lumbar Spine Instability," Neurosurgery, Vol. 47, No. 1, 2000, pp. 85-96. doi:10.1227/00006123-200007000-00019 
[8] K. Muramatsu, "Postoperative Magnetic Resonance Imaging of Lumbar Disc Herniation: Comparison of Microendoscopic Discectomy and Love's Method," Spine, Vol. 26, No. 14, 2001, pp. 1599-1605. doi:10.1097/00007632-200107150-00022

[9] M. Altay, "Treatment of Unstable Thoraco-Lumbar Junction Burst Fractures with Short-or Long-Segment Posterior Fixation in Magerl Type A Fractures," European Spine Journal, Vol. 16, No. 8, 2007, pp. 1145-1155. doi:10.1007/s00586-007-0310-5

[10] P. L. Sanderson, "Short Segment Fixation of Thoracolumbar Burst Fractures without Fusion," European Spine Journal, Vol. 8, No. 6, 1999, pp. 495-500. doi:10.1007/s005860050212

[11] X. Y. Wong, "Kyphosis Recurrence after Posterior ShortSegment Fixation in Thoraco-Lumbar Burst Fractures," Journal of Neurology, Spine, Vol. 8, No. 3, 2008, pp. 246-254.

[12] M. Aebi, et al., "AO ASIF Priciples in Spine Surgery," Springer-Verlag, Berlin, 1998, pp. 1-143. doi:10.1007/978-3-642-58824-2 1

[13] F. Denis, et al., "The Three Column Spine and Its Significance in the Classification of an Acute Thoracolumbar Spinal Injuries," Spine, Vol. 8, No. 8, 1983, pp. 817-831. doi:10.1097/00007632-198311000-00003

[14] R. Meves, "Correlation between Neurogical Deficit and Spinal Canal Compromise in 198 Patients with Thoracoloumbar and Lumbar Fractures," Spine, Vol. 30, No. 7, 2005 , pp. 787-791. doi:10.1097/01.brs.0000157482.80271.12

[15] S. P. Mohanty, "Does Neurological Recovery in ThoracoLumbar and Lumbar Burst Fractures Depend on the Extent of Canal Compromise," Spinal Cord, Vol. 40, No. 6, 2002, pp. 295-299. doi:10.1038/sj.sc.3101283

[16] A. R. Vaccaro, et al., "A New Classification of Thoracolumbar Injuries: The Importance of Injury Morphology, the Integrity of the Posterior Ligamentous Complex, and Neurologic Status," Spine, Vol. 30, No. 20, 2005, pp. 2325-2333. doi:10.1097/01.brs.0000182986.43345.cb

[17] R. A. Vaccaro, et al., "Surgical Decision Making for Unstable Thoracolumbar Spine Injuries," Journal of Spinal Disorders \& Techniques, Vol. 19, No. 1, 2006, pp. 1-10. doi:10.1097/01.bsd.0000180080.59559.45

[18] R. Yurec, B. Marre, A. Urzua, M. Munjin and M. A. Lecaros, "Residual Mobility of Instrumented and Non-Fused Segments in Thoracolumbar Spine Fractures," European Spine Journal, Vol. 15, No. 6, 2006, pp. 864-875. doi:10.1007/s00586-005-0939-x 